\title{
Nanoparticle Tracking Analysis of Gold Nanoparticles in Aqueous Media through an Inter-Laboratory Comparison
}

Sophie M. Briffa ${ }^{1}$, Jo Sullivan ${ }^{2}$, Agnieszka Siupa ${ }^{2}$, Pauline Carnell-Morris ${ }^{2}$, Michele Carboni ${ }^{2}$, Kerstin Jurkschat ${ }^{3}$, Ruud J. B. Peters ${ }^{4}$, Carolin Schultz ${ }^{5}$, Kang Hee Seol ${ }^{6}$, Sook-Jin Kwon ${ }^{6}$, Sehee Park ${ }^{7}$, Tae Hyun Yoon ${ }^{6,7}$, Colin Johnston ${ }^{3}$, Stephen Lofts $^{8}$, Eugenia Valsami-Jones ${ }^{1}$

${ }^{1}$ School of Geography, Earth and Environmental Sciences, University of Birmingham ${ }^{2}$ Malvern Panalytical ${ }^{3}$ Department of Materials, University of Oxford ${ }^{4}$ Wageningen Food Safety Research ${ }^{5}$ UK Centre for Ecology \& Hydrology ${ }^{6}$ Institute for Next Generation Material Design, Hanyang University ${ }^{7}$ Department of Chemistry, College of Natural Sciences, Hanyang University ${ }^{8}$ UK Centre for Ecology \& Hydrology

\section{Corresponding Author}

Sophie M. Briffa

BriffaSM@bham.ac.uk

\section{Citation}

Briffa, S.M., Sullivan, J., Siupa, A., Carnell-Morris, P., Carboni, M.,

Jurkschat, K., Peters, R.J.B., Schultz, C., Seol, K.H., Kwon, S.J., Park, S.,

Yoon, T.H., Johnston, C., Lofts, S.,

Valsami-Jones, E. Nanoparticle Tracking

Analysis of Gold Nanoparticles in

Aqueous Media through an Inter-

Laboratory Comparison. J. Vis. Exp.

(164), e61741, doi:10.3791/61741

(2020).

\section{Date Published}

October 20, 2020

DOI

$10.3791 / 61741$

\section{URL}

jove.com/video/61741

\section{Abstract}

In the field of nanotechnology, analytical characterization plays a vital role in understanding the behavior and toxicity of nanomaterials (NMs). Characterization needs to be thorough and the technique chosen should be well-suited to the property to be determined, the material being analyzed and the medium in which it is present. Furthermore, the instrument operation and methodology need to be well-developed and clearly understood by the user to avoid data collection errors. Any discrepancies in the applied method or procedure can lead to differences and poor reproducibility of obtained data. This paper aims to clarify the method to measure the hydrodynamic diameter of gold nanoparticles by means of Nanoparticle Tracking Analysis (NTA). This study was carried out as an inter-laboratory comparison (ILC) amongst seven different laboratories to validate the standard operating procedure's performance and reproducibility. The results obtained from this ILC study reveal the importance and benefits of detailed standard operating procedures (SOPs), best practice updates, user knowledge, and measurement automation.

\section{Introduction}

Nanomaterials (NMs) can vary in both physical and chemical characteristics that in turn influence their behavior, stability, and toxicity ${ }^{1,2,3,4,5}$. One of the major difficulties, when developing a thorough understanding of NM properties, hazards, and behaviors, is the ability to obtain reproducible information about physical and chemical nanomaterial characteristics. Examples of such physical properties include particle size and size distribution ${ }^{6,7,8}$. These are important 
parameters as they are a key aspect of the European Commission's (EC) definition of the term 'nano'9.

Achieving precise particle size measurements is also critical for many different industrial and research applications and processes in addition to understanding the fate and toxicity effects of $\mathrm{NMs}^{6,10}$. It is important to have well established methods capable of measuring accurately, reliably, and reproducibly the size of NMs. Furthermore, reported information should provide deep understanding of the technique used e.g., indicate the type of size parameter (e.g., actual size or hydrodynamic size) as well as the sample condition e.g., the specific medium in which the NM is present, and for the method to perform reliably in different media. In order to measure size, a number of techniques can be used, including electron microscopy (EM), dynamic light scattering (DLS), single particle inductively coupled plasma mass spectrometry (spICP-MS), differential centrifugal sedimentation (DCS), scanning probe microscopy (SPM), small-angle X-ray scattering (SAXS) and nanoparticle tracking analysis (NTA).

NTA is a relatively new technology which has been well advanced in recent years and has been shown to reliably measure the hydrodynamic diameter of spherical NMs in complex aqueous media such as those with environmental relevance, e.g., freshwater systems. The hydrodynamic diameter is 'the size of a hypothetical hard sphere that diffuses in the same fashion as that of the particle being measured'11; in practical terms and in aqueous media this describes a diameter larger than that of the particle itself, which also includes a layer of molecules (mostly water) held at the surface of the particle by weak electrostatic forces. The hydrodynamic diameter of a particle will vary in different media, getting smaller as the ionic strength of the media in which it is measured gets higher.

An additional important feature of the NTA technique is that it allows the analyst to achieve number-weighted size measurements, which are required in the context of the $\mathrm{EC}$ nanomaterial definition. High resolution, particle-by-particle analysis makes this technique less prone to interference caused by agglomerates or larger particles when present in a heterogeneous test sample with a high rate of particle throughput $^{10,12}$.

The measurement procedure consists of preparing a suitable suspension of the sample, which often requires sample dilution, followed by video recording of the particles' Brownian motion behavior and video analysis. From the sample chamber, a laser beam is passed, and the suspension particles in the path of the laser beam scatter light leading to their visualization using an optical microscope with a mounted camera. The camera captures a video file of the scattered laser light from the particles moving under Brownian motion. Many particles can be tracked individually to determine their diffusion coefficients and their hydrodynamic diameters can be calculated using the Stokes-Einstein equation: $d$ $=\mathrm{kT} / 3 \pi \mathrm{n} \mathrm{D}$ where $\mathrm{d}$ is the hydrodynamic diameter, $\mathrm{k}$ is the Boltzmann constant, $T$ is the temperature, $\eta$ is the viscosity and $D$ is the diffusion coefficient ${ }^{10}$. NTA can also be used to track the aggregation behavior of particles that are generally colloidally unstable (the particles must, however, be colloidally stable over the measurement time scale) $)^{13,14}$. NTA is an absolute method and no system calibration is required on the instrument used in this work. If users want to check the system performance this can be easily done by measuring size standard materials as frequently as wanted. 
The NTA instrument is easy to operate with quick analysis time (under $10 \mathrm{~min}$ per sample). For high quality measurements with good data repeatability and reproducibility, a number of factors should be considered in both sample preparation as well as in instrument operation. If such factors are not carefully considered, measurements on the same material across different laboratories and operators can be subject to unknown or poorly quantified uncertainties. During NP characterization, using best practice in-house developed SOPs does not always guarantee consistency with other laboratories, as shown by Roebben et al. for the DLS technique ${ }^{15}$.

In fact, an early (first round) NTA ILC between different laboratories, users and instruments revealed inconsistent results. One of the main issues was with the use of various older legacy instruments which had not had regular services or calibration checks, as well as differences in method interpretation. An NTA ILC study by Hole et al. found that with the absence of shared guidelines on how to use a system and prepare samples, variability across laboratories can be large even for relatively monodispersed samples ${ }^{16}$. This along with the results from the first round of the ILC highlights the need for good instrument maintenance as well as method training and well-developed standard operating procedures (SOPs). The latter act as a powerful tool to describe and document compliance with good practice. If well detailed, standard operating procedures (SOPs) can offer clarity, explanation, understanding, standardization, and quality assurance.

The recommendation for adopting an ILC study is, therefore, ideal for both developing and testing protocols ${ }^{16}$. The ILC exercise acted to validate this specific NTA SOP and hence introduced confidence and clarity into this specific nanomaterial risk assessment method. It involved three rounds. Round 1 analyzed $60 \mathrm{~nm}$ gold nanoparticles on each participant's own instruments before training. Round 2 involved analyzing $100 \mathrm{~nm}$ latex using a new instrument with common configuration as a simple test in order to determine that the instrument was set up correctly and the users had a good knowledge on how to use the instrument. Round 3 involved the analysis of $60 \mathrm{~nm}$ gold nanoparticles on the new instrument with common configuration, after training. Participants in the ILC came from seven different labs, all consortium members of the Horizon 2020 ACEnano project $^{17}$.

The aim of this article is to discuss the method and results from a third round of benchmarking for the NTA technology where $60 \mathrm{~nm}$ gold NPs were re-analyzed by seven partners following detailed training and SOP development. Comparison and reference to the results obtained in the first round of the ILC will also be made. All analyses from round 3 of ILC were carried out using the same instrument (see Table of Materials) of identical configuration supplied with a $405 \mathrm{~nm}$ laser and a high sensitivity sCMOS camera. Benchmarking assesses the performance of the technology on samples and hence leads to the development of 'best practice' protocols. Thus, this article also shares and makes the NTA method for the instrument used in this ILC available for the scientific community as it has been harmonized via conducting and evaluating the ILCs according to international standards.

\section{Protocol}

The methodology described here was used for the third round of the inter-laboratory comparisons. 


\section{Sample Preparation}

1. Filter water through a $0.02 \mu \mathrm{m}$ syringe filter. Water filtration is necessary to remove any contamination particles before using it for sample dilution.

2. To analyze a freshly prepared sample, dilute a sample of $60 \mathrm{~nm}$ gold colloid dispersion volumetrically by a factor of 50 in filtered ultrapure water. The suggested concentration for NTA analysis is $1 \times 10^{7}-1 \times 10^{9}$ particles per $\mathrm{mL}$.

\section{Performing the measurement}

1. Switching on the system

1. Connect the NTA instrument, syringe pump and the computer. Switch on the hardware and software. The associated software (see Table of Materials) ensures all hardware communications are running and that a live temperature readout is displayed.

2. Remove the laser module from the NTA and using a tissue and compressed air completely dry the glass surfaces and the low volume flow cell (LVFC) internal channels, tubing, and fluidic ports.

2. Priming the tubing

1. Rinse the inlet fluidic tubing with ultrapure water to remove any particles and reduce the likelihood of air bubbles that would interfere with measurements. For rinsing, the end of the inlet tubing inside the instrument casing is placed in a waste container.

2. Insert a $1 \mathrm{~mL}$ syringe (without needle) of filtered water into the Luer port and push $\sim 900 \mu \mathrm{L}$ of liquid through the inlet tubing as fast as the back pressure allows. Leave the syringe containing the remaining liquid attached to prevent any syphoning.

3. Syringe pump tubing connection

1. Assemble the LVFC onto the laser module to create the sample chamber as seen in Figure 1. Attach the outlet tubing to the right-hand side port of the LVFC. NOTE: The inlet and outlet tubing are different in diameters, with the inlet being smaller in diameter than the outlet. Swapping the inlet-outlet tubing connection may cause over pressuring the flow cell and leaking.

2. Disconnect the syringe from the inlet tubing and exchange for a new syringe containing $1 \mathrm{~mL}$ of filtered water, ensuring liquid-to-liquid contact. Connect the inlet tubing to the left port of the LVFC. Slowly introduce $\sim 500 \mu \mathrm{L}$ of fluid into the sample chamber. Take care to ensure no air bubbles are introduced during loading. The final tubing configuration is shown in Figure 2. 


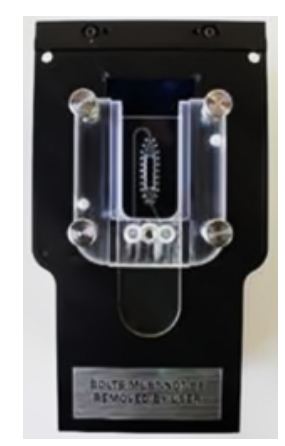

Figure 1: Low Volume Flow Cell assembly mounted on laser module.

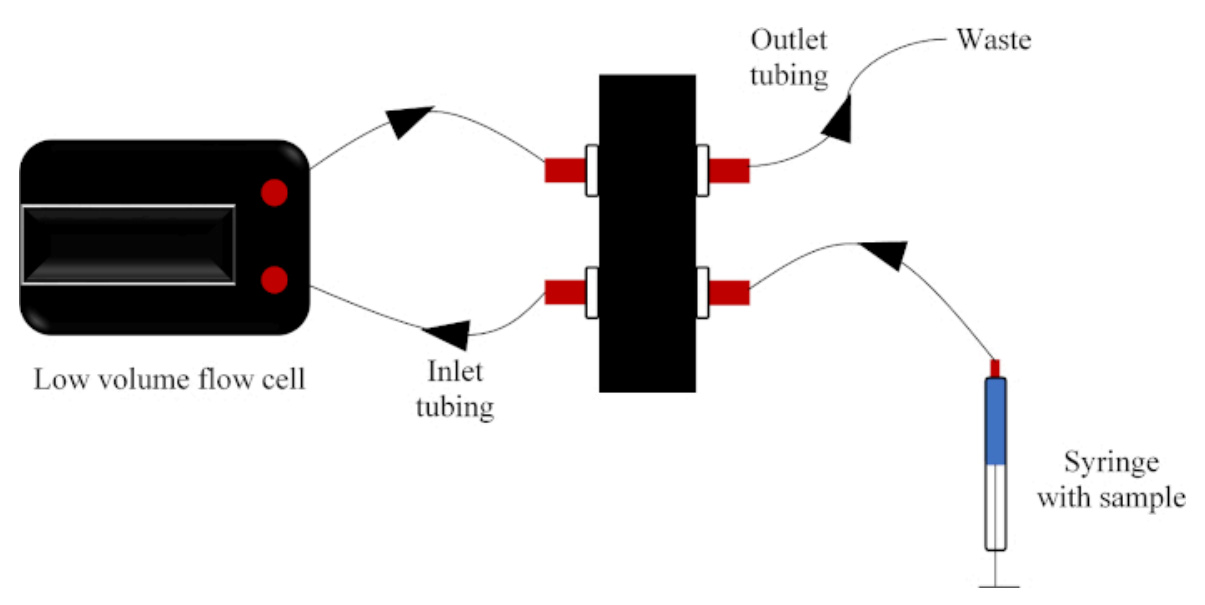

Figure 2: Low Volume Flow Cell tubing configuration. Please click here to view a larger version of this figure.

4. Laser module loading and system check

1. Insert the laser module with the water filled LVFC into the instrument and lock into place.

2. Place the syringe into the syringe pump cradle and secure. Initialize the camera by clicking on Start Camera in the software interface. In the Hardware tab of the interface, click on Scatter to move the reference position.

3. Set the camera level to 16 and adjust the focus manually to check the diluent for any particles. Adjust the field of view position by left clicking on the main viewing window and using the mouse to drag up and down to check for any particles. If there are more than three particles in the field of view, this implies a problem with the water purity or the cleaning process and, therefore, the cleaning process needs to be repeated or the water needs to be replaced or filtered.

4. Remove the laser module from the instrument.

5. Disconnect the syringe from the inlet tubing and replace it with a syringe full of air only. Slowly 
introduce the air into the sample chamber to remove the liquid inside. Remove the LVFC from the laser module and disconnect the tubings. Clean the glass surfaces of the LVFC and optical glass of the laser module with water and dry with a tissue and compressed air. Dry the tubing with compressed air. Reassemble the LVFC onto the laser module and connect the tubing, ready for sample loading.

NOTE: This step is not always required, however, in this case it was added as an extra precaution to further reduce any possible variation.

5. Loading sample

1. Repeat step 2.2.2. Connect a syringe containing $1 \mathrm{~mL}$ of the $60 \mathrm{~nm}$ gold nanoparticles dispersion made in step 1.1 to the Luer port. Slowly inject $750 \mu \mathrm{L}$ of the sample into the LVFC via the inlet tubing with the laser module viewed outside the instrument to ensure no bubbles are introduced.

2. Load the laser module back to the NTA instrument and initialize the camera by clicking on Start Camera in the software interface. In the Hardware tab of the interface, click Scatter to move to the reference focus position, check that this is set correctly to give a clear image of the particles.

3. Check that the field of view is set centrally with respect to the laser beam position. Adjust accordingly by left clicking on the main viewing window in the software and mouse dragging up and down.

4. Run the AutoSetup function to automatically optimize the focus and camera level ensuring that the optimal image quality is achieved.
NOTE: The automatic camera and focus parameters allow for more consistency amongst the different labs since this is user independent.

6. Sample analysis

1. Create a measurement script in Standard measurement, SOP tab, to obtain 5 repeat videos of $60 \mathrm{~s}$ under slow (particles should be passing across from one side of the screen to the other in approximately $10 \mathrm{~s}$ ) and constant flow (Supplementary File 1).

NOTE: Flow is recommended to ensure a better representation of the overall sample is presented for measurement. Precision and repeatability of concentration measurements are significantly improved when a slow flow is imparted on the sample to ensure that a greater number of new particles flow through the measurement zone and are analyzed during an experiment. The video length depends on the profile distribution and how variable it is over the analysis time. 5 videos of $60 \mathrm{~s}$ are considered as a typical measurement duration.

2. Set the experiment file name and location for the data and start the run. The analysis following the outlined procedure was carried out by the seven laboratories of the Horizon 2020 ACEnano project ${ }^{17}$.

\section{Data Analysis}

NOTE: All data analysis was done within the v 3.4 software (see Table of Materials), no additional manual conversions or calculations are used. The particle sizing data is presented in raw form as a histogram distribution and is calculated from the measured change in position of the particle using the Stokes-Einstein equation. The software determines the average distance moved by each particle in the $\mathrm{x}$ and $\mathrm{y}$ 
planes. This value allows the particle diffusion coefficient (D) to be determined from which, if the sample temperature $T$ and solvent viscosity $\eta$ are known, the equivalent spherical hydrodynamic radius, $\mathrm{R}_{\mathrm{H}}$, of the particles can be calculated. The temperature of the sample is automatically recorded by the NTA. The default sample viscosity used by the software is for water and is included in the measurement script shown above, though viscosity can be amended by the user when different sample diluents are used, either before or after the measurement is taken.

1. Set the detection threshold (DT) by dragging the slider bar or clicking the + and - buttons in the software under
Detection Threshold, which is the analysis parameter for optimal tracking of the visualized particles, between 2 and 20. Ensure that the DT value chosen identify and track as many visible particles as possible (marked automatically as red crosses on the software image screen).

1. As guidance for setting the detection threshold, the number of identified particles in an image should be in the range of approximately $30-80$ where no more than 10 red crosses should correspond to sites not considered to be particles by the observer. There should be no more than 5 blue crosses (indicative of noise) observed.
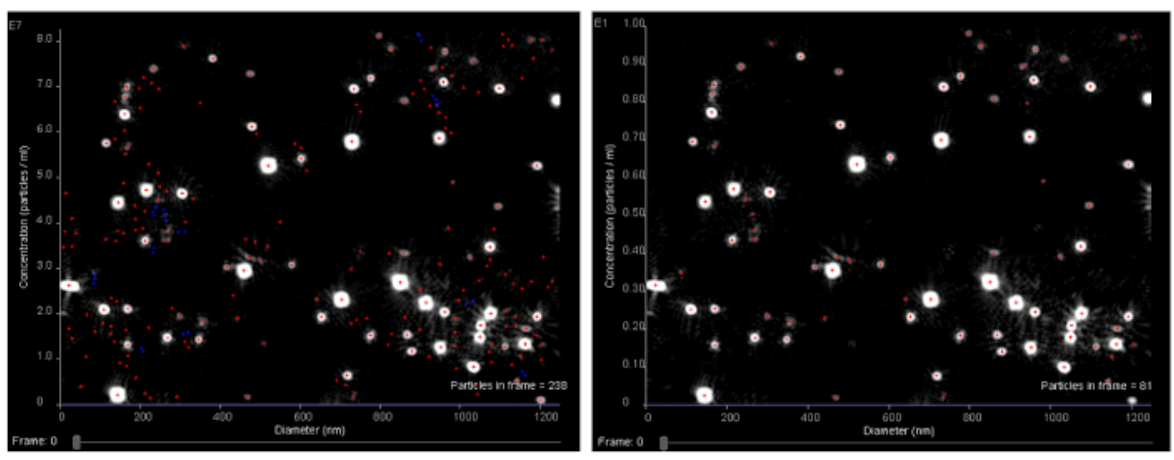

Figure 3: Threshold setting observations. A bad (left) and good (right) detection threshold setting observation. Please click here to view a larger version of this figure.

2. Automatically process the particle tracking analysis videos by pressing the Process button in the software. Leave all the processing parameters set to automatic and export the data as a .csv format results file with the full particle size distribution and additional metadata describing the measurement setup. To verify the measurement quality, look at the Analyze tab in the software or check the .csv output file for any warnings message or alerts. An example of the PDF results report is shown in Supplementary File 2.

3. Read the mode size results and the associated standard deviation from the PDF report.

NOTE: The mode size results were used to compare the sizes obtained amongst the seven laboratories and are shown and discussed in Section 5. 


\section{Cleaning and drying}

1. After use, flush the system thoroughly with clean water to remove all traces of sample from the tubing and optical surfaces. Cleaning effectiveness can be monitored by observation of the amount of particles in the field of view.

2. Remove the laser module from the NTA instrument.

3. Load a syringe of air through the system to empty the tubing and the LVFC.

\section{Representative Results}

The Round 1 ILC results using various NTA instrument configurations are shown in Figure 4. With the exception of Lab 6, the repeatability between the 5 capture repeats was good but several labs recorded a mode size higher than expected. Lab 6 results showed poor repeatability and a much higher mode size measured. After the investigation, it was found that the systems reporting biggest size variations were either not maintained as recommended or the analysis was affected by inconsistency in sample preparation whereby the dilution step can create variation caused by different pipetting equipment, user operation and technique, and/ or measurement set up including the flow cell not being clean, the wrong camera level being used, the image not being focused properly, and setting the analysis Detection Threshold incorrectly.

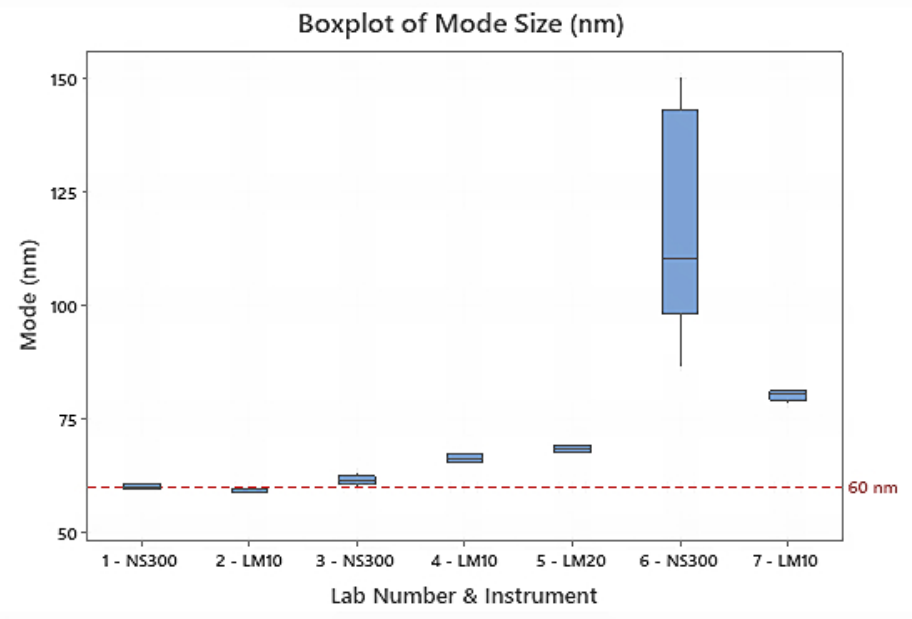

Figure 4: ILC Round 1 Mode size results. Mode size results from all NTA benchmarking partners for Round $160 \mathrm{~nm}$ gold nanoparticle dispersion carried out on different NTA instruments (as abbreviated in the $x$ axis).

The NTA result accuracy from Round 3 was improved by all the laboratories implementing the same SOP and instrument settings. The mode size results obtained for this ILC Round 3 can be seen in Figure 5. The average mode across all labs was $62.02 \pm 1.97 \mathrm{~nm}$. All measured results from Round 3 were more consistent than the first stage results with the results falling well within $10 \%$ of the $60.5 \mathrm{~nm}$ mean size for the batch as stated by the manufacturer. The coefficient of variation for the gold samples stated by the manufacturer was $\leq 8 \%$. 


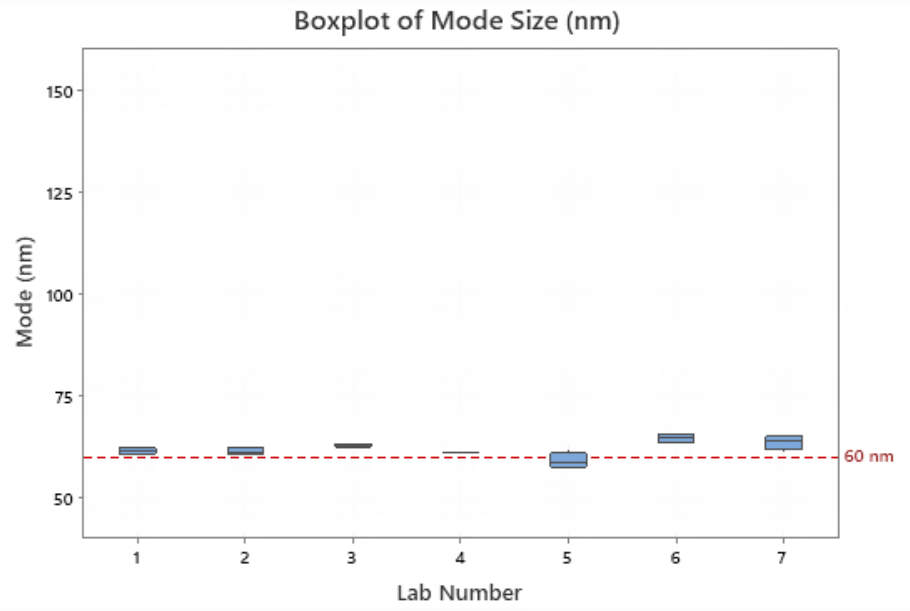

Figure 5: ILC Round 3 Mode size results. Mode size results from all NTA benchmarking partners for $60 \mathrm{~nm}$ gold ILC Round 3 analyzed on the same NTA instrument. The average mode across all labs was $62.02 \pm 1.97 \mathrm{~nm}$.

To verify the particle size, as provided by the manufacturer, a small number $(\mathrm{N}=82)$ of particles were analyzed by Transmission Electron Microscopy (TEM). Approximately 10 $\mu \mathrm{l}$ of the undiluted dispersion was drop cast on a carbon coated $\mathrm{Cu}$ TEM grid and dried in air before imaging in an analytical TEM at $200 \mathrm{kV}$. Images like Supplementary Figure 1 were taken from areas with minimal particle overlap and analyzed using a semi-automatic image analyses process. An automatic watershed method was applied to separate particles and artefacts of this process were excluded as well as on edge particles ${ }^{18}$. The mean diameter was calculated either as average from the major and minor axis $(61 \pm 7 \mathrm{~nm})$ or as a conversion from the measured area (62 $\pm 6 \mathrm{~nm}$ ) assuming spherical particles. Particles appear to be mostly spherical with an average aspect ratio of 1.1. The TEM results show a slightly higher diameter than the manufacturer value $(60.5 \mathrm{~nm})$ but are within the tolerance level. Additionally, there is a very good agreement with the NTA derived value of hydrodynamic diameter.
Supplementary Figure 1: TEM image of $60 \mathrm{~nm}$ Gold Nanoparticles. Please click here to download this figure.

Supplementary File 1: Measurement script. Please click here to download this file.

Supplementary File 2: Example of PDF results report. Please click here to download this file.

\section{Discussion}

The inconsistent results obtained from the Round 1 ILC highlighted the need for instrument health checks for older systems as well as the development of a more detailed SOP, the need for hands-on training and a better understanding of measurement and analysis settings so as to ensure more consistent results across the different labs. In fact, Hole et al. found that the absence of shared guidelines on how to use an NTA system and prepare samples resulted in variability across laboratories even for relatively monodispersed samples ${ }^{16}$. Therefore, all ILC participants attended a training workshop covering the best practices for 
the system operation and measurement conditions, as well as cleaning and maintenance guidance for the specific NTA instrument. All participants also performed measurements on the same instrument in their own labs for the subsequent ILC rounds. The procedure first involved a round that tested the system locally in each laboratory by running an ILC on latex standard samples (ILC Round 2), before being used by the partners to repeat the gold measurements (ILC Round 3). The aim of measuring these gold samples by means of NTA was to introduce confidence and clarity into nanomaterial risk assessment methods and practices needed to impact nanosafety guidance protocols.

NTA is a technique that can measure the hydrodynamic spherical equivalent diameter of particles and can be used for particle by particle, real-time visual analysis of polydispersed systems ranging from $10 \mathrm{~nm}-50 \mathrm{~nm}$, to approximately $1000 \mathrm{~nm}$ in size (depending on the sample properties and instrument configuration). Minimal sample preparation is required. Despite minimal sample preparation, this step is critical for the protocol and great care should be taken when diluting a sample and choosing a diluent. Shape can be a limiting factor with respect to NTA as spherical equivalent size measurements are obtained and non-spherical particles will have a less accurate size value.

For NTA technology, some result variation is always to be expected as only a representative sample is observed from the whole sample. Regardless, all results meet the ISO 19430 standard for particle sizing. The optimal concentration to provide is typically around $10^{8}$ particles $/ \mathrm{ml}$ within a 30-60 second analysis time. For samples with lower particle concentrations, longer analysis times will be required to ensure reproducible results. For samples containing a concentration of particles greater than $10^{9}$ particles $/ \mathrm{mL}$, there is a greater likelihood of tracking problems and samples will need to be diluted down to a suitable range for NTA measurement.

Overall the results from the $3^{\text {rd }}$ Round ILC show good reproducibility of gold nanoparticle measurements with NTA with increased accuracy and repeatability. All NTA measurements were carried out using the automatic camera level and focus settings to adjust the image, as selected by the Auto-Setup feature in the software. The camera level set by the software was very consistent, with a camera level of 10 or 11 being set in all cases showing that as expected, the more automation a process includes the more consistency is achieved. The sizing results were comparable to those obtained by the manufacturer by means of TEM indicating that the results were reproducible, however minimal differences are to be expected from different techniques since TEM does not determine the hydrodynamical diameter. The significant improvement in the consistency of results show the importance and benefits of instrument maintenance, detailed SOPs, best practice updates, user knowledge and applied measurement automation for NTA. In conclusion the ILC validated this specific NTA SOP and hence introduced confidence and clarity into this specific nanomaterial risk assessment method.

\section{Disclosures}

The author Jo Sullivan, Agnieszka Siupa, Pauline CarnellMorris and Michele Carboni are employees at Malvern Panalytical Ltd. that manufactures instruments used in this article.

\section{Acknowledgments}

The authors acknowledge financial support from H2020 funded project: ACEnano (Grant Agreement no 720952). 
This work was also partially supported by the International Cooperative R\&D Program funded by the Ministry of Trade, Industry, and Energy of Korea (grant number N053100009, "Horizon2020 Kor-EU collaborative R\&BD on ACEnano Toolbox") which enabled participation of the Korean partners in the consortium of Horizon 2020 ACEnano Project.

\section{References}

1. Thwala, M., Musee, N., Sikhwivhilu, L., Wepener, V. The oxidative toxicity of $\mathrm{Ag}$ and $\mathrm{ZnO}$ nanoparticles towards the aquatic plant Spirodela punctuta and the role of testing media parameters. Environmental Science-Processes and Impacts. 15 (10), 1830-1843 (2013).

2. Lowry, G. V., Gregory, K. B., Apte, S. C., Lead, J. R. Transformations of nanomaterials in the environment. Environmental Science \& Technology. 46 (13), 6893-6899 (2012).

3. Auffan, M. et al. Towards a definition of inorganic nanoparticles from an environmental, health and safety perspective. Nature Nanotechnology. 4 (10), 634-641 (2009).

4. Valsami-Jones, E., Lynch, I. How safe are nanomaterials? Science. 350 (6259), 388-389 (2015).

5. Briffa, S. M., Nasser, F., Valsami-Jones, E., Lynch, I. Uptake and impacts of polyvinylpyrrolidone (PVP) capped metal oxide nanoparticles on Daphnia magna: role of core composition and acquired corona. Environmental Science: Nano. 5, 1745-1756 (2018).

6. Hassellöv, M. a. R. K. Analysis and Characterization of Manufactured Nanoparticles in Aquatic Environments. Environmental and Human Health Impacts of Nanotechnology. 211-266, John Wiley \& Sons, Ltd. (2009).
7. Hoet, P., Brüske-hohlfeld, I., Salata, O. Nanoparticles and known and unknown health risks. Journal of Nanobiotechnology. 2 (1), 12 (2004).

8. Briffa, S. M. et al. Thermal transformations of manufactured nanomaterials as a proposed proxy for ageing. Environmental Science: Nano. 5, 1618-1627 (2018)

9. European Commission. Nanotechnology. <http:// ec.europa.eu/nanotechnology/policies_en.html > (2011).

10. Kestens, V., Bozatzidis, V., De Temmerman, P.J., Ramaye, Y., Roebben, G. Validation of a particle tracking analysis method for the size determination of nano- and microparticles. Journal of Nanoparticle Research. 19 (8), 271 (2017).

11. Malvern. Dynamic Light Scattering - common terms defined. <http://www.malvern.com/en/support/resourcecenter/Whitepapers/

WP111214DLSTermsDefined.aspx> (2015).

12. Walker, J. G. Improved nano-particle tracking analysis. Measurement Science and Technology. 23 (6), 065605 (2012)

13. Panalytical, M. Using NTA to Study Aggregation Behavior of Liposome-Protein Complexes. <https://www.newsmedical.net/whitepaper/20161125/Using-NTA-to-StudyAggregation-Behavior-of-Liposome-ProteinComplexes.aspx> (2019).

14. Filipe, V., Hawe, A., Jiskoot, W. Critical evaluation of nanoparticle tracking analysis (NTA) by NanoSight for the measurement of nanoparticles and protein aggregates. Pharmaceutical Research. 27 (5), 796-810 (2010).

15. Roebben, G. et al. Interlaboratory comparison of size and surface charge measurements on nanoparticles prior 
to biological impact assessment. Journal of Nanoparticle Research. 13 (7), 2675 (2011).

16. Hole, P. et al. Interlaboratory comparison of size measurements on nanoparticles using nanoparticle tracking analysis (NTA). Journal of Nanoparticle Research: An Interdisciplinary Forum for Nanoscale Science and Technology. 15 (12), 2101-2101 (2013).

17. ACEnano. Analytical and Characterisation Excellence in nanomaterial risk assessment: A tiered approach. <https://cordis.europa.eu/project/id/720952> (2019).

18. Soille, P., Vincent, L. Determining watersheds in digital pictures via flooding simulations. Vol. 1360 VC, SPIE. (1990).

Copyright $\odot 2020$ JoVE Creative Commons Attribution-NonCommercial-NoDerivs 3.0 Unsorted

jove.com

October $2020 \cdot 164 \cdot$ e61741 Page 12 of 12 